\title{
Dyslipidemias and Related Factors in Brazilian Adolescents from Rural and Urban Areas
}

\author{
Daniela Alves Silva ${ }^{1}$, Sylvia do Carmo Castro Franceschini ${ }^{1}$, Juliana Faria de Novaes ${ }^{1}$, \\ Ricardo Henrique Silva Santos ${ }^{2}$, Silvia Eloiza Priore ${ }^{1}$ \\ ${ }^{1}$ Departamento de Nutrição e Saúde, Universidade Federal de Viçosa, Viçosa, Brazil \\ ${ }^{2}$ Departamento de Fitotecnia, Universidade Federal de Viçosa, Viçosa, Brazil \\ Email: danielasilvauai@yahoo.com.br
}

Received 28 March 2014; revised 2 May 2014; accepted 10 May 2014

Copyright (C) 2014 by authors and Scientific Research Publishing Inc.

This work is licensed under the Creative Commons Attribution International License (CC BY). http://creativecommons.org/licenses/by/4.0/

c) (i) Open Access

\section{Abstract}

This study aimed to evaluate the prevalence of dyslipidemias and related factors, in Brazilian adolescents from rural and urban areas. This is a cross-sectional study with 182 adolescents aged 10 to 13 years, students, residing in the rural and urban area of Brazil; grouped according to age, gender and socioeconomic class. Sexual maturation, physical activity, blood pressure, nutritional status and body composition were evaluated. Total cholesterol, LDL, HDL and serum triglycerides were dosed. The food frequency questionnaire was applied, quantifying energy, carbohydrates, proteins, lipids, full fat and fibers. Bivariate and multiple analyses were carried out, by logistic regression. It was verified that $84.6 \%(n=154)$ adolescents presented some lipid profile alteration. There was no difference in the lipid profile between adolescents for residence place. However, as for gender, it was noticed that girls presented higher levels of total cholesterol and HDL $(p<0.05)$. In addition, adolescents with excess weight and body fat showed lower HDL and higher triglycerides values compared with eutrophic and with those with appropriate body fat percentage $(p<$ $0.05)$. In the multiple analyses, it was observed that gender and signs of sexual maturation were associated with total cholesterol; fiber intake remained associated with HDL and signs of sexual maturation with LDL $(p<0.05)$. For triglycerides the related factors were gender, nutritional status and protein intake $(p<0.05)$. The elevated prevalence of dyslipidemias found between adolescents from rural and urban areas reinforces the importance of monitoring these alterations and information as for the related risk factors, with programs for correction of changeable factors.

\section{Keywords}

Dyslipidemias, Adolescents, Lifestyle, Puberty 


\section{Introduction}

Cardiovascular diseases (CVD) represent one of the main causes of morbimortality in Brazil and in the world [1]. It is treated as a multifactorial disease caused by the interaction of genetic factors, excess weight and body fat, high arterial blood pressure, dyslipidemias, inadequate eating habit, sedentarism and tobaccoism [2] [3].

The probability of CVD incidence is proportional to the number of risk factors [4]. Dyslipidemias stand out as determining factors of these diseases, being a great public health concern [5]-[7]. They are characterized by abnormal concentrations of lipid or lipoproteins serum levels [8], sometimes identified in young individuals as children and adolescents [1].

In growth and developmental stages, the influences of sexual hormones must also be considered, as they are responsible for physiologically increasing lipid serum levels, especially in early puberty [2].

The importance of tracking the risk factors in childhood and adolescence for planning early interventions stands out, aiming at the maintenance of the identified risk situation in adult life [7]. Besides, as these are resulting of multifactorial etiology, there might be differences according to the characteristics of the studied population, such as the area of residence, urban or rural [9].

The present study aimed to evaluate the prevalence of dyslipidemias and related factors, in Brazilian adolescents residing in rural and urban areas.

\section{Methodology}

\subsection{Study Delineation}

This is an observational cross-sectional study with 182 public school adolescent aged 10 to 13 years, from rural and urban areas of Viçosa (MG, Brazil). The adolescents reside in the school area (rural or urban) and had not received nutritional follow-up in the last six months.

Initially, all students from the rural area in the interest age group $(n=132)$ were contacted; however, 110 were eligible, out of which 91 (82.7\%) participated in the study. Considering age, gender and socioeconomic class, these students were grouped with those residing in the urban area at 1:1 ratio, totalizing 182 adolescents to the final sample.

The project was approved by the Research Ethics Committee of the Universidade Federal de Viçosa, MG, Brazil (process number 054/2011) and the parents/caregivers signed a free and informed consent term.

\subsection{Socioeconomic Evaluation}

For grouping and socioeconomic classification, the questionnaire of the Brazilian Association of Research Companies [10] which considers the presence of consumer goods and education level of the head of family was used. To help with the grouping of adolescents from rural and urban areas, classes B and C1 and C2, D and E were grouped together.

\subsection{Evaluation of Physical Activity Practice}

The short version [11] of the International Physical Activity Questionnaire (IPAQ) was applied categorizing adolescents as inactive (sedentary and irregularly active) and active (active and very active).

\subsection{Anthropometrical and Physical Composition Evaluation}

According to techniques recommended by the World Health Organization (WHO) [12], weight was verified in a Kratos $^{\circledR}$ electronic scale with $150 \mathrm{~kg}$ capacity and 50 g precision (Cotia, SP, Brazil), and the stature was evaluated, in duplicate, using an Alturexata ${ }^{\circledR}$ vertical anthropometer, 2 meters extension (Belo Horizonte, MG, Brazil), using the average of obtained values as the individual's stature. From the weight (in kilogram) and stature (in meters) measures, the body mass index (BMI) was calculated, classified by age and gender, according to the anthropometrical references of the WHO [13], using z-scores, calculated in the software WHO Antroplus.

The body composition was estimated (BARBOSA, 2006), using equipment Dual X-ray Absorptiometry (DEXA), obtaining body fat percentage data (BF\%), classified according to Lohman [14].

\subsection{Blood Pressure}

The blood pressure was checked in a digital automatic inflation appliance, recommended by the Brazilian Car- 
diology Society (Sociedade Brasileira de Cardiologia), following the recommendations of the Brazilian Society of Hypertension (Sociedade Brasileira de Hipertensão) [15]. The definition of the blood pressure percentile was carried out considering age, gender and adolescent stature percentile, evaluated by WHO [13], classifying bordering values as unhealthy also.

\subsection{Evaluation of Sexual Maturation}

During the anthropometrical evaluation, sexual maturation was characterized, observing the presence of secondary sexual characteristics such as axillary hair, in boys and girls, facial hair, in boys and development of breasts and menarche, in girls [16]. According to this evaluation, sexual maturation signs were classified as present or absent.

\subsection{Dietetic Evaluation}

The frequent food questionnaire was applied with adolescents in the presence of parents/caregivers, using domestic measure utensils to help in the portioning. The preparations were detailed, standardizing the recipes [17]. Dietetic analyses were carried out using Diet Pro 5.5i software (Viçosa, MG, Brazil), quantifying energy, carbohydrates, lipids, trans fat and fibers.

The caloric intake was compared with the estimate energy requirement (EER) value, classifying adolescents as above or below this value. For evaluation of carbohydrates and lipid consumption, the recommendation of the AMDR (Acceptable Macronutrient Distribution Ranges) [18] was considered. For protein, the cut point $1 \mathrm{~g} / \mathrm{kg}$ weight [19], trans fat, up to 7\% of the total energetic value [2] and the AI (Adequate Intake) value for fibers [18] were adopted.

\subsection{Biochemical Evaluation}

The biochemical evaluation was carried out after 12 hours of fast, dosing total cholesterol (TC), HDL (High Density Lipoprotein), LDL (Low Density Lipoprotein) and triglycerides. For characterization of the lipid profile, the recommendations of the Sociedade Brasileira de Cardiologia (Brazilian Society of Cardiology) [2] were used, considering as unhealthy values classified as bordering and increased for total cholesterol, LDL and triglycerides, as well as reduced values for HDL. Individuals who presented alteration in at least one of the mentioned parameters were considered dyslipidemia bearers.

\subsection{Statistical Analyses}

The database was analyzed in duplicate using Microsoftware Excel, Epi Info 6.04, Sigma Statistic for Windows and Stata 9.1 programs. The Kolmogorov-Sminorv test was carried out to check the normality of data, parametric or non-parametric tests for paired samples, comparison and correlation and Chi-square test to verify associations.

The odds ratio (OR) and respective 95\% confidence intervals for associations between dependent (total cholesterol, HDL, LDL and triglycerides) and independent (sociodemographic condition, signs of sexual maturation, nutritional status, physical fat and lifestyle) variables were estimated.

Variables which in bivariate analysis were associated with dependent variables with significance level lower than 0.20 were included in the multiple logistic model. A model was prepared for each studied variable, maintaining independent variables that presented association with the dependant variable with significance level lower than 0.05 in the final model.

\section{Results}

The sampling was constituted by 182 adolescents from rural and urban areas, 62.6\% $(\mathrm{n}=114)$ boys, aged 10.02 to 13.95 years, with median and mean \pm SD of 11.13 and $11.42 \pm 1.0$ years, respectively.

Regarding the lipid profile, the most altered parameters between adolescents were mean values for total cholesterol and LDL (Figure 1), with overall mean of $161.1 \pm 28.9$ and $95.3 \pm 25.8 \mathrm{mg} / \mathrm{dL}$, respectively. Median values (minimum - maximum) for HDL and triglycerides were 50.0 (23.0 - 117.0) and 64.0 (14.0 - 248.0) $\mathrm{mg} / \mathrm{dL}$, respectively. It was verified that $84.6 \%(\mathrm{n}=154)$ adolescents presented some lipid profile alteration.

When total cholesterol, HDL, LDL and triglycerides levels were compared between adolescents, no difference was found according to the residence place. However, when gender was considered, girls presented higher 


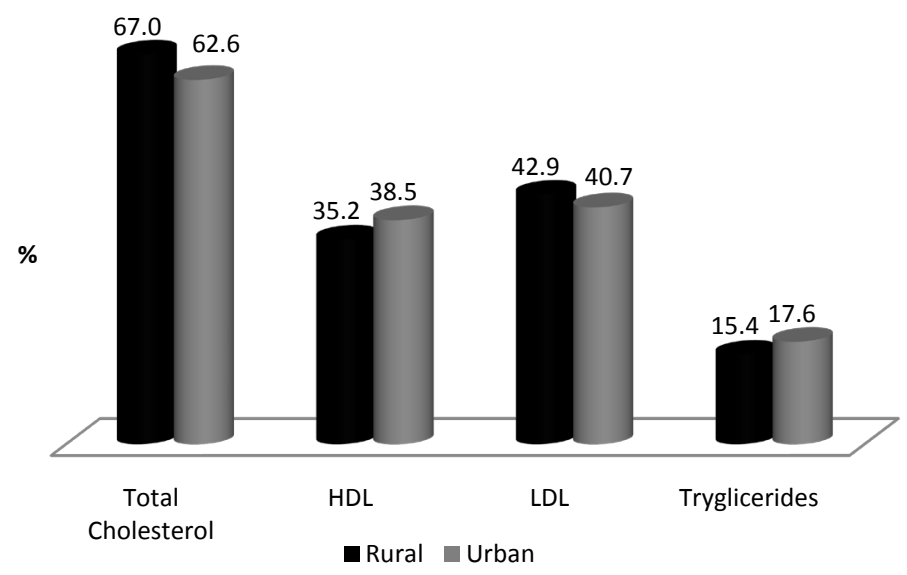

Figure 1. Lipid profile of Brazilian adolescents residing in rural and urban areas.

total cholesterol and HDL levels (Table 1).

Of the evaluated individuals, $21.4 \%(n=39)$ were classified as excess weight and $33.5 \%(n=61)$ as excess body fat. These adolescents presented lower HDL and higher triglycerides values comparing with eutrophic adolescents with appropriate body fat percentage. Besides, those with excess body fat presented higher total cholesterol and LDL serum levels (Table 1).

It is emphasized that both for rural and urban area adolescents, means and medians of the total cholesterol values were higher than $150 \mathrm{mg} / \mathrm{dL}$, both for nutritional status and body fat in adequate or excessive conditions (Table 1).

Table 2 presents results of bivariate analyses for total cholesterol, HDL, LDL and triglycerides levels concerning sociodemographic, sexual maturation, physical activity, nutritional status, body fat, blood pressure and dietetic intake characteristics.

Altered total cholesterol was associated with gender and signs of sexual maturation. Male adolescents presented lower probability of alteration in this parameter (OR, odds ratio, 0.47 ; IC, interval confidence $95 \%$ : -1.4 - 0.15; $\mathrm{p}=0.02$ ). Presence of sexual maturation signs represented protection regarding the altered total cholesterol (OR: 0.33; IC 95\%: 0.13 - 0.79; p = 0.01) (Table 2).

Regarding the HDL fraction, it was associated with age and signs of sexual maturation variables. Adolescents with age above median (11.1 years) were more protected as for this alteration (OR: 0.45; IC 95\%: 0.24 - 0.83; $p$ $=0.01)$. The presence of sexual maturation signs showed 76\% less chance of low HDL values [(1 - 0.24)*100]. In addition, gender $(\mathrm{p}=0.14)$ and lipid intake $(\mathrm{p}=0.08)$ variables were included in the multiple analysis (Table 2$)$.

As for the LDL fraction, association was observed with gender and fiber intake. Male adolescents presented twice as high probability of altered LDL (IC 95\%: 1.05 - 3.88; $\mathrm{p}=0.03$ ) and those with fiber intake below the recommended level also had more chance of alteration in this parameter (OR: 1.96; IC 95\%: $1.06-3.63$; $\mathrm{p}=$ $0.03)$. Additionally, physical activity $(p=0.15)$, nutritional status $(p=0.12)$ and energetic intake $(p=0.09)$ variables were included in the multiple analysis.

Altered triglyceride was associated with gender, nutritional status, body fat percentage, protein and full fat intake. Male adolescents presented $56 \%$ less chance of alteration in this parameter [(1 - 0.44)*100]. Excess weight and body fat increased the chances for this biochemical alteration (OR: 3.15; IC 95\%: $1.37-7.20$; $=0.006$ and OR: 2.97; IC 95\%: 1.24 - 7.10; $\mathrm{p}=0.01$, respectively). Both protein and saturated fat intake above the recommended levels were associated with protection (OR: 0.17; IC 95\%: $0.07-0.44$; p < 0.001), (OR: 0.35: IC 95\%: $0.14-0.87 ; p=0.02)$, respectively. In addition, physical activity $(\mathrm{p}=0.13)$ and energetic intake $(\mathrm{p}=0.19)$ were included in the multiple analysis.

It was verified that gender $(\mathrm{p}=0.03)$ and sexual maturation signs $(\mathrm{p}=0.02)$ showed association with total cholesterol in the multiple analyses.

Regarding HDL, fiber intake was the variable that maintained associated with it; adolescents with fiber intake below the recommended level presented 1.97 times higher chance to have alteration in this biochemical parameter (Table 3). 
Table 1. Comparison of lipid profile according to place of residence, gender, nutritional status and body fat of adolescents from Brazil.

\begin{tabular}{|c|c|c|c|c|}
\hline & $\begin{array}{l}\text { Total cholesterol } \\
(\mathrm{mg} / \mathrm{dL})^{*}\end{array}$ & $\begin{array}{c}\text { HDL } \\
(\mathrm{mg} / \mathrm{dL})^{* *}\end{array}$ & $\begin{array}{c}\text { LDL } \\
(\mathrm{mg} / \mathrm{dL})^{*}\end{array}$ & $\begin{array}{c}\text { Triglycerides } \\
(\mathrm{mg} / \mathrm{dL})^{* *}\end{array}$ \\
\hline & $\begin{array}{c}\text { Mean } \pm \text { SD } \\
\text { Median (Min - Max) }\end{array}$ & $\begin{array}{c}\text { Mean } \pm \text { SD } \\
\text { Median (Min - Max) }\end{array}$ & $\begin{array}{c}\text { Mean } \pm \text { SD } \\
\text { Median (Min - Max) }\end{array}$ & $\begin{array}{c}\text { Mean } \pm \text { SD } \\
\text { Median (Min - Max) }\end{array}$ \\
\hline \multicolumn{5}{|l|}{ Area of residence } \\
\hline Rural & $\begin{array}{c}162.1 \pm 30.5 \\
161.0(92.0-234.0)\end{array}$ & $\begin{array}{c}52.5 \pm 15.5 \\
51.0(23.0-117.0)\end{array}$ & $\begin{array}{c}95.8 \pm 24.4 \\
93.6(35.0-166.6)\end{array}$ & $\begin{array}{c}69.1 \pm 36.1 \\
60.0(25.0-228.0)\end{array}$ \\
\hline Urban & $\begin{array}{c}160.1 \pm 27.3 \\
162.0(99.0-210.0)\end{array}$ & $\begin{array}{c}50.2 \pm 12.0 \\
49.0(29.0-106.0)\end{array}$ & $\begin{array}{c}94.9 \pm 23.4 \\
95.6(46.2-140.4)\end{array}$ & $\begin{array}{c}74.7 \pm 36.1 \\
68.0(14.0-248.0)\end{array}$ \\
\hline $\mathbf{p}$ & 0.66 & 0.21 & 0.82 & 0.19 \\
\hline \multicolumn{5}{|l|}{ Gender } \\
\hline Girls & $\begin{array}{c}166.7 \pm 28.7 \\
168.5(92.0-234.0)\end{array}$ & $\begin{array}{c}53.2 \pm 12.5 \\
52.0(23.0-97.0)\end{array}$ & $\begin{array}{c}97.8 \pm 27.7 \\
100.8(35.0-166.6)\end{array}$ & $\begin{array}{c}78.2 \pm 39.1 \\
66.5(30.0-228.0)\end{array}$ \\
\hline Boys & $\begin{array}{c}157.7 \pm 28.6 \\
158.0(99.0-224.0)\end{array}$ & $\begin{array}{c}50.2 \pm 14.5 \\
46.5(29.0-117.0)\end{array}$ & $\begin{array}{c}93.9 \pm 23.3 \\
91.7(46.2-154.8)\end{array}$ & $\begin{array}{c}68.1 \pm 33.8 \\
63.0(14.0-248.0)\end{array}$ \\
\hline $\mathbf{p}$ & 0.04 & 0.04 & 0.27 & 0.08 \\
\hline \multicolumn{5}{|l|}{ Nutritional status } \\
\hline Eutrophic and low weight & $\begin{array}{c}160.1 \pm 29.3 \\
160.0(92.0-224.0)\end{array}$ & $\begin{array}{c}53.2 \pm 15.5 \\
51.0(29.0-117.0)\end{array}$ & $\begin{array}{c}94.0 \pm 23.0 \\
90.2(35.0-154.8)\end{array}$ & $\begin{array}{c}64.2 \pm 25.0 \\
60.5(25.0-167.0)\end{array}$ \\
\hline Excess weight & $\begin{array}{c}166.1 \pm 30.5 \\
166.0(107.0-234.0)\end{array}$ & $\begin{array}{c}45.5 \pm 8.7 \\
45.0(23.0-66.0)\end{array}$ & $\begin{array}{c}100.9 \pm 26.9 \\
103.0(47.4-166.6)\end{array}$ & $\begin{array}{c}98.3 \pm 51.6 \\
94.0(27.0-248.0)\end{array}$ \\
\hline $\mathbf{p}$ & 0.29 & 0.01 & 0.14 & $<0.001$ \\
\hline \multicolumn{5}{|l|}{ Body fat } \\
\hline Ideal and low & $\begin{array}{c}157.8 \pm 29.7 \\
158.0(92.0-224.0)\end{array}$ & $\begin{array}{c}52.8 \pm 13.8 \\
52.0(29.0-106.0)\end{array}$ & $\begin{array}{c}92.6 \pm 23.4 \\
89.8(35.0-154.8)\end{array}$ & $\begin{array}{c}62.2 \pm 23.5 \\
60.0(14.0-139.0)\end{array}$ \\
\hline Excess & $\begin{array}{c}167.6 \pm 26.1 \\
159.0(107.0-234.0)\end{array}$ & $\begin{array}{c}48.5 \pm 13.6 \\
46.0(23.0-117.0)\end{array}$ & $\begin{array}{c}100.8 \pm 24.0 \\
102.2(47.4-166.6)\end{array}$ & $\begin{array}{c}91.3 \pm 47.5 \\
84.0(25.0-248.0)\end{array}$ \\
\hline $\mathbf{p}$ & 0.03 & 0.02 & 0.02 & $<0.001$ \\
\hline
\end{tabular}

SD, Standard Deviation; Med, Median; Min, Minimum; Max, Maximum; HDL, High Density Lipoprotein; LDL, Low Density Lipoprotein. ${ }^{{ }^{*}}$ Paired t-test; ${ }^{\text {a** }}$ Wilcoxon's test; ${ }^{\text {b* }}$ Student t-test; ${ }^{\text {b** }}$ Mann Whitney’s test $\mathrm{p}<0.05$.

Table 2. Prevalence of alterations in lipid profile and gross odds ratio (95\% interval confidence) according to sociodemographic, sexual maturation signs, nutritional status, body fat and lifestyle variables of rural and urban adolescents from Brazil.

\begin{tabular}{|c|c|c|c|c|c|c|c|c|c|c|c|c|}
\hline & \multicolumn{3}{|c|}{ Total Cholesterol } & \multicolumn{3}{|c|}{ HDL } & \multicolumn{3}{|c|}{ LDL } & \multicolumn{3}{|c|}{ Triglycerides } \\
\hline & Altered (\%) & $\begin{array}{l}\text { Gross OR } \\
\text { (IC 95\%) }\end{array}$ & $\mathrm{p}$ & Altered (\%) & $\begin{array}{l}\text { Gross OR } \\
\text { (IC 95\%) }\end{array}$ & $\mathrm{p}$ & Altered (\%) & $\begin{array}{l}\text { Gross OR } \\
\text { (IC 95\%) }\end{array}$ & $\mathrm{p}$ & Altered (\%) & $\begin{array}{l}\text { Gross OR } \\
\text { (IC 95\%) }\end{array}$ & $\mathrm{p}$ \\
\hline Área & & & & & & & & & & & & \\
\hline Rural & $61(67.0)$ & 1.0 & & $32(35.2)$ & 1.0 & & 39 (42.9) & 1.0 & & $14(15.4)$ & 1.0 & \\
\hline Urban & $57(62.6)$ & $\begin{array}{c}1.21 \\
(0.65-2.23)\end{array}$ & 0.53 & 35 (38.5) & $\begin{array}{c}0.86 \\
(0.47-1.58)\end{array}$ & 0.64 & 37 (40.7) & $\begin{array}{c}1.13 \\
(0.62-2.05)\end{array}$ & 0.67 & 16 (17.6) & $\begin{array}{c}0.35 \\
(0.38-1.86)\end{array}$ & 0.69 \\
\hline Gender & & & & & & & & & & & & \\
\hline Girls & $52(76.5)$ & 1.0 & & $18(26.5)$ & 1.0 & & $34(50.0)$ & 1.0 & & $16(23.5)$ & 1.0 & \\
\hline Boys & $66(57.9)$ & $\begin{array}{c}0.47 \\
(-1.4-0.15)\end{array}$ & 0.02 & 49 (43.0) & $\begin{array}{c}2.02 \\
(1.05-3.88)\end{array}$ & 0.03 & $42(36.8)$ & $\begin{array}{c}0.63 \\
(0.34-1.16)\end{array}$ & 0.14 & 14 (12.3) & $\begin{array}{c}0.44 \\
(0.20-0.97)\end{array}$ & 0.04 \\
\hline Age & & & & & & & & & & & & \\
\hline$\leq 11.1$ years & $63(69.2)$ & 1.0 & & $31(34.1)$ & 1.0 & & $47(51.6)$ & 1.0 & & $18(19.8)$ & 1.0 & \\
\hline$>11.1$ years & $55(60.4)$ & $\begin{array}{c}0.67 \\
(0.36-1.25)\end{array}$ & 0.21 & 36 (39.6) & $\begin{array}{c}1.26 \\
(0.69-2.31)\end{array}$ & 0.44 & 29 (31.9) & $\begin{array}{c}0.45 \\
(0.24-0.83)\end{array}$ & 0.01 & 12 (13.2) & $\begin{array}{c}0.61 \\
(0.27-1.36)\end{array}$ & 0.23 \\
\hline $\begin{array}{c}\text { Sexual } \\
\text { maturation } \\
\text { signs }\end{array}$ & & & & & & & & & & & & \\
\hline Absent & $108(68.4)$ & 1.0 & & $56(35.4)$ & 1.0 & & $72(45.6)$ & 1.0 & & $27(17.1)$ & 1.0 & \\
\hline Present & $10(41.7)$ & $\begin{array}{c}0.33 \\
(0.13-0.79)\end{array}$ & 0.01 & $11(45.8)$ & $\begin{array}{c}1.54 \\
(0.64-3.66)\end{array}$ & 0.33 & $4(16.6)$ & $\begin{array}{c}0.24 \\
(0.08-0.76)\end{array}$ & 0.01 & $3(12.5)$ & $\begin{array}{c}0.69 \\
(0.19-2.48)\end{array}$ & 0.57 \\
\hline
\end{tabular}




\section{Continued}

\begin{tabular}{|c|c|c|c|c|c|c|c|c|c|c|c|c|}
\hline $\begin{array}{l}\text { Physical } \\
\text { atividade }\end{array}$ & & & & & & & & & & & & \\
\hline Yes & $96(65.7)$ & 1.0 & & $50(34.3)$ & 1.0 & & $63(43.2)$ & 1.0 & & $21(14.4)$ & 1.0 & \\
\hline No & $22(61.1)$ & 0.81 & 0.60 & $17(47.2)$ & 1.71 & 0.15 & $13(36.1)$ & 0.72 & 0.41 & $9(25.0)$ & 1.98 & 0.13 \\
\hline & & $(0.38-1.73)$ & & & $(0.82-3.59)$ & & & $(0.34-1.54)$ & & & $(0.81-4.80)$ & \\
\hline $\begin{array}{c}\text { Nutritional } \\
\text { status }\end{array}$ & & & & & & & & & & & & \\
\hline $\begin{array}{l}\text { Eutrophic and } \\
\text { low weight }\end{array}$ & 91 (63.6) & 1.0 & & 49 (34.3) & 1.0 & & 54 (37.8) & 1.0 & & 15 (10.5) & 1.0 & \\
\hline Excess weight & $27(69.2)$ & 1.19 & 0.57 & $18(46.2)$ & 1.20 & 0.55 & $22(56.4)$ & 1.60 & 0.12 & 15 (38.5) & 3.15 & 0.006 \\
\hline & & $(0.64-2.20)$ & & & $(0.65-2.20)$ & & & $(0.87-2.91)$ & & & $(1.37-7.20)$ & \\
\hline $\begin{array}{c}\text { Body fat \% } \\
\text { Low and }\end{array}$ & & & & & & & & & & & & \\
\hline Adequate & $72(59.5)$ & 1.0 & 0.66 & 40 (33.1) & 1.0 & 0.53 & $45(37.2)$ & 1.0 & & $10(8.3)$ & 1.0 & \\
\hline Excess & $46(75.4)$ & 1.14 & & 27 (44.3) & 1.21 & & $31(50.8)$ & 1.21 & 0.53 & $20(32.8)$ & 2.97 & 0.01 \\
\hline & & $(0.62-2.10)$ & & & $(0.66-2.21)$ & & & $(0.66-2.21)$ & & & $(1.24-7.1)$ & \\
\hline $\begin{array}{c}\text { Arterial } \\
\text { blood } \\
\text { pressure }\end{array}$ & & & & & & & & & & & & \\
\hline Normal & $115(65.0)$ & 1.0 & & 66 (37.3) & 1.0 & & 75 (42.4) & 1.0 & & 30 (16.9) & 1.0 & \\
\hline Altered & $3(60.0)$ & 0.80 & 0.81 & $1(20.0)$ & 0.42 & 0.44 & $1(20.0)$ & 0.44 & 0.49 & - & - & - \\
\hline & & $(0.13-4.96)$ & & & $(0.04-3.04)$ & & & $(0.04-4.40)$ & & & & \\
\hline $\begin{array}{c}\text { Energetic } \\
\text { Intake }\end{array}$ & & & & & & & & & & & & \\
\hline Below & 53 (63.8) & 1.0 & & $36(43.4)$ & 1.0 & & 33 (39.8) & 1.0 & & $17(20.5)$ & 1.0 & \\
\hline Above & $65(65.7)$ & 1.08 & 0.80 & 31 (31.3) & 0.59 & 0.09 & 43 (43.3) & 1.26 & 0.54 & 13 (13.1) & 0.58 & 0.19 \\
\hline & & $(0.58-1.99)$ & & & $(0.32-1.09)$ & & & $(0.66-2.18)$ & & & $(0.26-1.29)$ & \\
\hline $\begin{array}{c}\text { Carbohydrate } \\
\text { intake }\end{array}$ & & & & & & & & & & & & \\
\hline Adequate & $96(66.7)$ & 1.0 & & 53 (36.8) & 1.0 & & 63 (43.8) & 1.0 & & 22 (15.3) & 1.0 & \\
\hline Below & 22 (18.7) & 0.73 & 0.35 & 14 (20.9) & 0.87 & 0.70 & $13(17.1)$ & 1.05 & 0.88 & $8(26.7)$ & 1.08 & 0.85 \\
\hline & & $(0.37-1.41)$ & & & $(0.44-1.71)$ & & & $(0.55-2.02)$ & & & $(0.46-2.56)$ & \\
\hline $\begin{array}{l}\text { Protein } \\
\text { Intake }\end{array}$ & & & & & & & & & & & & \\
\hline Below & 17 (68.0) & 1.0 & & $11(44.0)$ & 1.0 & & $10(40.0)$ & 1.0 & & $11(44.0)$ & 1.0 & \\
\hline Above & $101(64.3)$ & 0.84 & 0.72 & $56(35.7)$ & 0.70 & 0.42 & $66(42.0)$ & 1.11 & 0.81 & 19 (12.1) & 0.17 & 0.00 \\
\hline & & $(0.34-2.09)$ & & & $(0.30-1.65)$ & & & $(0.47-2.63)$ & & & $(0.07-0.44)$ & \\
\hline $\begin{array}{l}\text { Lipid } \\
\text { intake }\end{array}$ & & & & & & & & & & & & \\
\hline Adequate & 70 (64.2) & 1.0 & & 41 (37.6) & 1.0 & & 40 (36.7) & 1.0 & & 16 (14.7) & 1.0 & \\
\hline Above & 48 (65.8) & 1.06 & 0.83 & $26(35.6)$ & 0.91 & 0.78 & 36 (49.3) & 1.7 & 0.08 & $14(19.2)$ & 1.37 & 0.42 \\
\hline & & $(0.57-1.99)$ & & & $(0.49-1.69)$ & & & $(0.92-3.11)$ & & & $(0.62-3.03)$ & \\
\hline $\begin{array}{c}\text { Saturated fat } \\
\text { intake }\end{array}$ & & & & & & & & & & & & \\
\hline Adequate & $16(55.2)$ & 1.0 & & $12(41.4)$ & 1.0 & & 12 (41.4) & 1.0 & & $9(31.0)$ & 1.0 & \\
\hline Above & $102(66.6)$ & 1.62 & 0.24 & 55 (35.9) & 0.79 & 0.58 & $64(41.8)$ & 1.04 & 0.92 & $21(13.7)$ & 0.35 & 0.02 \\
\hline & & $(0.72-3.63)$ & & & $(0.35-1.78)$ & & & $(0.46-2.33)$ & & & $(0.14-0.87)$ & \\
\hline $\begin{array}{l}\text { Fiber } \\
\text { intake }\end{array}$ & & & & & & & & & & & & \\
\hline Adequate & 55 (63.2) & 1.0 & & $25(32.5)$ & 1.0 & & 35 (40.2) & 1.0 & & & 1.0 & \\
\hline Below & 63 (66.3) & 1.14 & 0.66 & $42(44.2)$ & 1.96 & 0.03 & $41(43.2)$ & 1.08 & 0.79 & $13(14.9)$ & 1.24 & 0.59 \\
\hline & & $(0.62-2.10)$ & & & $(1.06-3.63)$ & & & $(0.59-1.96)$ & & $17(17.9)$ & $(0.56-2.73)$ & \\
\hline
\end{tabular}

HDL, High Density Lipoprotein; LDL, Low Density Lipoprotein; OR, Odds Ratio; IC, Interval Confidence. Values in bold represent p $<0.05$. 
Table 3. Multiple analysis final results of association between sociodemographic, sexual maturation signs, nutritional status, body fat, lifestyle conditions and serum lipid profile of rural and urban adolescents from Brazil.

\begin{tabular}{|c|c|c|c|c|c|c|c|c|}
\hline & \multicolumn{2}{|c|}{ Total Cholesterol } & \multicolumn{2}{|c|}{ HDL } & \multicolumn{2}{|c|}{ LDL } & \multicolumn{2}{|c|}{ Triglycerides } \\
\hline & $\begin{array}{c}\text { OR } \\
\text { (IC 95\%) }\end{array}$ & $\mathrm{p}$ & $\begin{array}{c}\text { OR } \\
\text { (IC 95\%) }\end{array}$ & $\mathrm{p}$ & $\begin{array}{c}\text { OR } \\
\text { (IC 95\%) }\end{array}$ & $\mathrm{p}$ & $\begin{array}{c}\text { OR } \\
\text { (IC 95\%) }\end{array}$ & $\mathrm{p}$ \\
\hline \multicolumn{9}{|l|}{ Gender } \\
\hline Girls & 1.0 & & & & & & 1.0 & \\
\hline Boys & $\begin{array}{c}0.46 \\
(0.23-0.91)\end{array}$ & 0.03 & - & - & - & - & $\begin{array}{c}0.41 \\
(0.17-0.97)\end{array}$ & 0.04 \\
\hline \multicolumn{9}{|l|}{$\begin{array}{c}\text { Sexual } \\
\text { maturation signs }\end{array}$} \\
\hline Absent & 1.0 & & - & - & 1.0 & & - & - \\
\hline Present & $\begin{array}{c}0.36 \\
(0.15-0.88)\end{array}$ & 0.02 & & & $\begin{array}{c}0.24 \\
(0.1-0.8)\end{array}$ & 0.01 & & \\
\hline \multicolumn{9}{|l|}{ Nutritional status } \\
\hline $\begin{array}{l}\text { Eutrophic and low weight } \\
\text { Excess weight }\end{array}$ & - & - & - & - & - & - & $\begin{array}{c}1.0 \\
3.34 \\
(\mathbf{1 . 3 8}-\mathbf{8 . 0 9})\end{array}$ & 0.01 \\
\hline \multicolumn{9}{|l|}{ Protein intake } \\
\hline $\begin{array}{l}\text { Below } \\
\text { Above }\end{array}$ & - & - & - & - & - & - & $\begin{array}{c}1.0 \\
0.18 \\
(0.07-0.50)\end{array}$ & $<0.001$ \\
\hline \multicolumn{9}{|l|}{ fiber intake } \\
\hline $\begin{array}{l}\text { Adequate } \\
\text { Below }\end{array}$ & - & - & $\begin{array}{c}1.0 \\
1.96 \\
(\mathbf{1 . 1}-\mathbf{3 . 6})\end{array}$ & 0.03 & - & - & - & - \\
\hline
\end{tabular}

OR, Odds Ratio; IC, Interval Confidence. Values in bold represent $\mathrm{p}<0.05$.

As for LDL, adolescents presenting sexual maturation signs, showed 76\% lower alteration chances comparing with those who did not present these signs [(1 - 0.24)*100] (Table 3).

Factors independently associated with triglycerides alterations were gender $(\mathrm{p}=0.04)$, nutritional status $(\mathrm{p}=$ 0.01 ) and protein intake above the recommended level ( $<$ 0.001) (Table 3).

It is worth emphasizing that gender and sexual maturation were associated in two of the elaborated models (Table 3).

\section{Discussion}

The prevalence of dyslipidemia found in this study was elevated, similar to a study with children and adolescents with excess weight, residing in Campina Grande city (PB, Brazil), in which 85.3\% exhibited dyslipidemia $[1]$.

Although students from Pernambuco (PE, Brazil) aged 10 to 14 years demonstrated inferior prevalence, more than half (63.8\%) [20] presented at least one lipid profile alteration. In this sense, investigations on this risk factor between adolescents are relevant, as they represent a disturbing reality.

The absence of lipid profile difference in adolescents, according to area of residence observed in the present study, suggests that other factors inherent to this life stage could be involved with this lipid alteration However, in a study comparing the prevalence of dyslipidemias between 3121 Mexican students aged 12 to 16 years residing in rural and urban areas, higher prevalence was more frequent in adolescents of the rural area $(\mathrm{p}<0.001)$ [9].

The differences between studies can be due to the area of Viçosa (MG, Brazil) having atypical rural population comparing with other places, with rural population representing only $6.8 \%$ the total population [21]. Moreover, the studies were carried out in different countries, with age groups of different stages of adolescence.

The fact that girls presented higher lipid serum levels than boys might be related to sexual hormone alterations due to growth and development, especially in the initial stage of adolescence [2].

In this sense, in a study carried out with children and adolescents from Pernambuco, only total cholesterol and 
triglycerides levels were more elevated in girls ( $p=0.03$ and $p=0.04$, respectively) [5]. In Mexico, adolescent girls presented higher serum levels of total cholesterol, LDL, triglycerides and HDL than those of boys [9]. Similar result was found between American adolescents aged 12 to 19 years, for the two last mentioned parameters [22]. However, some studies did not identify differences in the lipid profile between genders [6] [20] [23].

Excess weight is an important risk factor for the development of cardiovascular alterations, predisposing individuals to the increase in total cholesterol, LDL, and triglycerides serum levels and to HDL reduction [24].

In the present study, excess weight represented higher chance of triglycerides alteration. Likewise, Pereira et $a l$. [25] evaluated children and adolescents aged 2 to 19 years in rural and urban areas of Itapetininga (SP, Brazil) and verified that individuals with excess weight presented greater risk of total cholesterol and triglycerides increase. Conversely, in the study of Grillo et al. [6], obesity resulted in 3.27 higher risk for HDL alteration, not significant for the other evaluated lipid parameters. This demonstrates the close relation between excess weight and dyslipidemias.

In addition, Romaldini et al. [26] studied the risk factors for atherosclerosis in 109 children and adolescents with family history of premature coronary artery disease and identified lipid alterations in $57.1 \%$ (16/28) of those with excess weight (obese and overweight) and in 32\% (26/55) of those with normal or low weight. Adolescents with excess weight presented twice higher chances (IC 95\% $=1.16-6.81$ ) of dislipidemia, however there was no association between dyslipidemia and family income, parental schooling and practice of physical activity variables.

Likewise, when evaluating clinical-metabolic alterations in obese and non obese adolescents grouped according to gender, color, age, sexual maturation stage and schooling, lower HDL levels and superior triglycerides levels among obese adolescents were observed [24].

In Tunisia, the prevalence of hypercholesterolemia among school children with excess weight was superior comparing with eutrophic children $(14.6 \%$ and $7.1 \%$, respectively, $\mathrm{p}<0.001)$. Moreover, girls presented more total cholesterol and LDL alterations $(\mathrm{p}<0.001)$ [7].

The sexual maturation process described by biological alterations may influence individual [16] lipid and lipoprotein blood levels [2]. Considering this variable in the lipid profile evaluation of 1846 urban Mexican adolescents aged 12 to 16 years, it was identified that during advancing of the pubertal stage, evaluated by selfevaluation according to Tanner, boys were negatively associated with total cholesterol, LDL and triglycerides [27].

It should be emphasized that besides the biological influence in question, adolescents presenting puberty signs experience higher environment interference in the definition of habits, including eating and physical activity and this will influence the lipid profile [20]. However a limitation of this study was not considered sexual maturation of the annealing of the sample.

The present study reinforces the importance of dietetic factors as determinant of plasmatic lipids, having in mind that inadequate fiber intake was associated with HDL, and proteins above the recommended level, with triglycerides.

In a study with adolescents aged 12 to 19 years who participated in the National Health and Nutrition Examination Survey (NHANES) 1999-2002, it was verified that to each increase in the amount of fiber intake, there was a $20 \%$ reduction in the incidence of metabolic syndrome, which corresponds to the set of cardiovascular risk factors, including dyslipidemias (adjusted OR $=0.83$, IC 95\% $0.68-1.00$ and $p=0.043$ ) [22].

Protein ingestion above the recommended level reduced the risk of triglycerides alteration. The opposite was expected, given that the main sources of proteins in the diet are foods of animal origin which also have higher amounts of fat in their composition. However, the source of ingested protein was not investigated in this study.

The modern lifestyle characterized by inadequate offer of caloric foods, with high contents of full fat, trans fat, cholesterol, simple carbohydrates and salt, may lead to an increased consumption and consequently to the development of risk factors like excess weight, high arterial blood pressure, diabetes and dyslipidemias [3].

\section{Conclusions}

The elevated prevalence of dyslipidemias found between adolescents of urban and rural areas reinforces the importance of monitoring this alteration in early ages. It is necessary to consider the influence of sexual maturation and gender, besides planning actions for adjustment of the nutritional status and the adopted lifestyle habits.

Actions taken during childhood and adolescence allow the reduction in the incidence of cardiovascular disease and its consequences in adulthood, favoring the promotion of health of these individuals at all stages of life. 


\section{Conflict of Interest}

The authors declare that there is no conflict of interest.

\section{References}

[1] Ramos, A.T., Carvalho, D.F., Gonzaga, N.C., Cardoso, A.S., Noronha, J.A.F. and Cardoso, M.A.A. (2011) Perfil lipídico em crianças e adolescentes com excesso de peso. Revista Brasileira de Crescimento Desenvolvimento Humano, 21, 780-788.

[2] Sociedade Brasileira de Cardiologia (2005) I diretriz de prevenção da aterosclerose na infância e na adolescência. Arquivos Brasileiros de Cardiologia, 85, 1-36.

[3] Mendes, G.A., Martinez, T.L., Izar, M.C., Amancio, O.M., Novo, N.F., Matheus, S.C., et al. (2006) Perfil lipídico e efeitos da orientação nutricional em adolescentes com história familiar de doença arterial coronariana prematura. Arquivos Brasileiros de Cardiologia, 86, 361-365. http://dx.doi.org/10.1590/S0066-782X2006000500006

[4] Gerber, Z.R.S. and Zielinsky, P. (1997) Fatores de risco de aterosclerose na infância. Um estudo epidemiológico. Arquivos Brasileiros de Cardiologia, 69, 231-236. http://dx.doi.org/10.1590/S0066-782X1997001000002

[5] Franca, E. and Alves, J.G.B. (2006) Dislipidemia entre crianças e adolescentes de Pernambuco. Arquivos Brasileiros de Cardiologia, 87, 722-727. http://dx.doi.org/10.1590/S0066-782X2006001900007

[6] Forti, N., Issa, J., Diament, J. and Giannini, S.D. (1998) Dislipidemia em criança e adolescentes. Bases para a terapêutica. Arquivos Brasileiros de Cardiologia, 71, 807-810. http://dx.doi.org/10.1590/S0066-782X1998001200012

[7] Grillo, L.P., Crispim, S.P., Siebert, N.A., Andrade, A.T.W., Rossi, A. and Campos, I.C. (2005) Perfil lipídico e obesidade em escolares de baixa renda. Revista Brasileira de Epidemiologia, 8, 75-81. http://dx.doi.org/10.1590/S1415-790X2005000100009

[8] Harrabi, I., Ghannem, H., Gaha, R., Hochlaf, M., Limam, K. and Essoussi, A.S. (2005) Epidemiology of Dyslipidemia among School Children in Sousse, Tunisia. Diabetes Metabolism, 31, 285-289. http://dx.doi.org/10.1016/S1262-3636(07)70195-7

[9] Yamamoto-Kimura, L., Posadas-Romero, C., Posada-Sánchez, R., Zamora-González, J., Cardoso-Saldana, G. and Ramirez, I.M. (2006) Prevalence and Interrelations of Cardiovascular Risk Factors in Urban and Rural Mexican Adolescent. Journal of Adolescent Health, 38, 591-598. http://dx.doi.org/10.1016/j.jadohealth.2005.04.004

[10] Associação Brasileira de Empresas de Pesquisa (ABEP) (2011) Critério de classificação econômica Brasil. http://www.abep.org/novo/Content.aspx?SectionID=84

[11] Celafisc (2011) Questionário internacional de atividade física-IPAQ (versão curta). http://www.celafiscs.institucional.ws/65/questionarios.html

[12] World Health Organization (WHO) (1995) Phisycal Status: The Use and Interpretation of Anthropometry. WHO, Geneva, Technnical Report Series, 854. http://helid.digicollection.org/en/d/Jh0211e/

[13] World Health Organization (WHO) (2011) Growth Reference 5 - 19 Anos. http://www.who.int/growthref/en/

[14] Lohman, T.G. (1992) Assessing Fat Distribution. Advances in Body Composition Assessment: Current Issues in Exercise Science. Human Kinects, Illinois, 57-63.

[15] Sociedade Brasileira de Hipertensão (2010) VI Diretrizes Brasileiras de Hipertensão Arterial. Arquivos Brasileiros de Cardiologia, 95, 1-51.

[16] Silva, D.A., Gontijo, C.A., Crizel, M.M. and Priore, S.E. (2010) Avaliação da maturação sexual de adolescentes: Autoavaliação versus exame clínico. In: Priore, S.E., Oliveira, R.M.S., Faria, E.R., Franceschini, S.C.C. and Pereira, P.F., Eds., Nutrição e Saúde na Adolescência, Rubio, Rio de Janeiro, 167-172.

[17] Pinheiro, A.B.V., Lacerda, E.M.A., Benzecry, E.H., Gomes, M.C.S. and Costa, V.M. (2005) Tabela para avaliação do

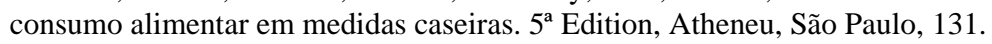

[18] Leal, G.V.S. (2008) Consumo alimentar, estado nutricional e nível de atividade física de adolescentes do Projeto Ilhabela-SP. Dissertação de Mestrado, Universidade de São Paulo, São Paulo, 131.

[19] Institute of Medicine (2002) Dietary References Intakes for Energy, Carboidrate, Fiber, Fat, Fatty Acids, Cholesterol, Protein and Amino Acids. The National Academy Press, Washington DC.

[20] Organizacion Mundial de La Salud (1985) Necessidades de energia y de proteínas. FAO/OMS/ONU, Geneva, 220. (Série de informes técnicos 724).

[21] Pereira, P.B., Arruda, I.K.G., Cavalcanti, A.M.T.S. and Diniz, A.S. (2010) Perfil em escolares de Recife-PE. Arquivos Brasileiros de Cardiologia, 95, 606-613. http://dx.doi.org/10.1590/S0066-782X2010005000136

[22] Seki, M., Seki, M.O., Lima, A.D., Onishi, M.H.O., Seki, M.O. and Oliveira, L.A.G. (2001) Estudo do perfil lipídico de 
crianças e jovens até 19 anos de idade. Jornal Brasileiro de Patologia, 37, 247-251.

[23] Carvalho, D.F., Paiva, A.A., Melo, A.S.O., Ramos, A.T., Medeiros, J.S., Medeiros, C.C.M. and Cardoso, M.A.A. (2007) Perfil lipídico e estado nutricional de adolescentes. Revista Brasileira de Epidemiologia, 10, 491-498. http://dx.doi.org/10.1590/S1415-790X2007000400007

[24] Posadas-Sánchez, R., Posadas-Romero, C., Zamora-González, J., Mendoza-Pérez, E., Cardoso-Saldana, G. and YamamotoKimura, L. (2007) Lipid and Lipoprotein Profiles and Prevalence of Dyslipidemia in Mexican Adolescents. Metabolism Clinical and Experimental, 56, 1666-1672. http://dx.doi.org/10.1016/j.metabol.2007.07.009

[25] Carlson, J.J., Eisenmann, J.C., Norman, G.J., Ortiz, K.A. and Young, P.C. (2011) Dietary Fiber and Nutrient Density Are Inversely Associated with the Metabolic Syndrome in US Adolescents. American Dietetic Association, 111, 16881695. http://dx.doi.org/10.1016/j.jada.2011.08.008

[26] Carneiro, J.R.I., Kushnir, M.C., Clemente, E.L.S., Brandão, M.G. and Gomes, M.B. (2000) Obesidade na adolescência: Fator de risco para complicações clínico-metabólicas. Arquivos Brasileiros de Endocrinologia e Metabologia, 44, 390396. http://dx.doi.org/10.1590/S0004-27302000000500005

[27] Pereira, A., Guedes, A.D., Verreschi, I.T.N., Santos, R.D. and Martinez, T.L.R. (2009) A obesidade e sua associação com os demais fatores de risco cardiovascular em escolares de Itapetininga, Brasil. Arquivos Brasileiros de Cardiologia, 93, 253-260. http://dx.doi.org/10.1590/S0066-782X2009000900009

\section{Abbreviations}

CVD: Cardiovascular Diseases

IPAQ: International Physical Activity Questionnaire

WHO: World Health Organization (WHO)

DEXA: Dual X-ray Absorptiometry

EER: Estimate Energy Requirement

AMDR: Acceptable Macronutrient Distribution Ranges

AI: Adequate Intake

HDL: High Density Lipoprotein

LDL: Low Density Lipoprotein

OR: Odds Ratio

IC: Interval Confidence

SD: Standard Deviation

MED: Median

MIN: Minimum

MAX: Maximum 